\title{
Global exact boundary controllability of a class of quasilinear hyperbolic systems of conservation laws II
}

\author{
De-Xing Kong* and Hui Yao \\ Department of Mathematics \\ Shanghai Jiao Tong University \\ Shanghai 200030, China
}

\begin{abstract}
In this paper, by a new constructive method, the authors reprove the global exact boundary controllability of a class of quasilinear hyperbolic systems of conservation laws with linearly degenerate fields. It is shown that the system with nonlinear boundary conditions is globally exactly boundary controllable in the class of piecewise $C^{1}$ functions. In particular, the authors give the optimal control time of the system. Finally, a new application is also given.
\end{abstract}

Keywords: Quasilinear hyperbolic system; Conservation laws; Global exact boundary controllability; Cauchy problem, Goursat problem, Classical discontinuous solution, Contact discontinuity, Optimal control time.

2000 Mathematics Subject Classification: 93C20, 49J20.

\footnotetext{
${ }^{*}$ Corresponding author.
} 


\section{Introduction}

Consider the following quasilinear system in a form of conservation laws

$$
\left\{\begin{array}{l}
u_{t}+f(u, v)_{x}=0 \\
v_{t}+g(u, v)_{x}=0
\end{array}\right.
$$

where $u=u(t, x)$ and $v=v(t, x)$ are unknown functions and $f, g \in C^{2}(\mathcal{N})$ for some closed bounded domain $\mathcal{N}$ in $\mathbb{R}^{2}$. Let $F=(f, g)^{T}$ and

$$
\nabla F(U)=\left(\begin{array}{ll}
f_{u} & f_{v} \\
g_{u} & g_{v}
\end{array}\right),
$$

where $U=(u, v)$. We assume that

$\left(H_{1}\right) \quad$ On the domain $\mathcal{N}$ under consideration, system (1.1) is strongly strictly hyperbolic, i.e., for any given $U \in \mathcal{N}, \nabla F(U)$ has two distinct real eigenvalues $\lambda_{1}(U), \lambda_{2}(U)$ :

$$
\lambda_{1}(U)<0<\lambda_{2}(U), \quad \forall U \in \mathcal{N} .
$$

Let $\vec{l}_{i}(U)=\left(l_{i 1}(U), l_{i 2}(U)\right)$ (resp. $\left.\vec{r}_{i}(U)=\left(r_{i 1}(U), r_{i 2}(U)\right)^{T}\right)$ be a left (resp. right) eigenvector corresponding to $\lambda_{i}(U)(i=1,2)$ :

$$
\vec{l}_{i}(U) \nabla F(U)=\lambda_{i}(U) \vec{l}_{i}(U) \quad\left(\operatorname{resp} . \nabla F(U) \vec{r}_{i}(U)=\lambda_{i}(U) \vec{r}_{i}(U)\right) .
$$

$\left(H_{2}\right)$ System (1.1) is linearly degenerate:

$$
\nabla \lambda_{i}(U) \cdot \vec{r}_{i}(U) \equiv 0 \quad(i=1,2), \quad \forall U \in \mathcal{N}
$$

$\left(H_{3}\right) \quad$ For any given real number $a$, let $H_{i}^{a} \triangleq\left\{U \mid \lambda_{i}(U)=a\right\}(i=1,2)$. For any given $U_{1}, U_{2}$ $\in H_{i}^{a}$, there exists a $C^{1}$ curve segment $U=U(\tau)\left(\tau \in\left[\tau_{1}, \tau_{2}\right]\right)$ in $\mathcal{N}$ such that

$$
U\left(\tau_{j}\right)=U_{j} \quad(j=1,2) \quad \text { and } \quad U(\tau) \in H_{i}^{a}, \quad \forall \tau \in\left[\tau_{1}, \tau_{2}\right]
$$

and

$$
\nabla \lambda_{i}(U(\tau)) \neq 0 \quad \forall \tau \in\left[\tau_{1}, \tau_{2}\right] \quad(i=1,2) .
$$

$\left(H_{4}\right)$ There are global Riemann invariants for the system (1.1)

$$
R_{1}=R_{1}(U), \quad R_{2}=R_{2}(U)
$$

Remark 1.1 Any quasilinear hyperbolic system with two unknown functions

$$
\frac{\partial u_{i}}{\partial t}+\sum_{j=1}^{2} a_{i j}\left(u_{1}, u_{2}\right) \frac{\partial u_{j}}{\partial x}=0 \quad(i=1,2)
$$


can always be reduced to a system with the diagonal form at least in a local domain. This means that for any quasilinear hyperbolic system with two unknown functions, the local Riemann invariants always exist. On the other hand, many physical systems (for example, the system of isentropic gas) always possess global Riemann invariants.

By the assumptions $\left(H_{2}\right)$ and $\left(H_{4}\right)$, in the Riemann invariants, (1.1) can be rewritten as

$$
\left\{\begin{array}{c}
\frac{\partial R_{1}}{\partial t}+\mu_{1}\left(R_{2}\right) \frac{\partial R_{1}}{\partial x}=0 \\
\frac{\partial R_{2}}{\partial t}+\mu_{2}\left(R_{1}\right) \frac{\partial R_{2}}{\partial x}=0
\end{array}\right.
$$

where

$$
\mu_{1}\left(R_{2}(U)\right)=\lambda_{1}(U) \quad \text { and } \quad \mu_{2}\left(R_{1}(U)\right)=\lambda_{2}(U)
$$

Recently, Kong [5] investigates the following Exact Boundary Control Problem for the system (1.1). Consider system (1.1) posed on the domain

$$
\mathcal{D}=\{(t, x) \mid t \geq 0, \quad-1 \leq x \leq 1\}
$$

with the following nonlinear boundary conditions

$$
\begin{array}{ll}
B_{1}(u, v, t)+h_{1}(t)=0 & \text { at } x=-1, \\
B_{2}(u, v, t)+h_{2}(t)=0 & \text { at } x=1
\end{array}
$$

and the initial data

$$
t=0:(u, v)= \begin{cases}\left(u_{0}^{-}(x), v_{0}^{-}(x)\right), & \forall x \in[-1,0] \\ \left(u_{0}^{+}(x), v_{0}^{+}(x)\right), & \forall x \in[0,1]\end{cases}
$$

where $B_{i}(u, v, t)$ are given smooth functions, $\left(u_{0}^{-}(x), v_{0}^{-}(x)\right) \in \mathcal{N}$ and $\left(u_{0}^{+}(x), v_{0}^{+}(x)\right) \in \mathcal{N}$ are $C^{1}$ vector functions, defined for $x \in[-1,0]$ and $x \in[0,1]$ respectively, satisfying

$$
\left(u_{0}^{-}(0), v_{0}^{-}(0)\right) \neq\left(u_{0}^{+}(0), v_{0}^{+}(0)\right) .
$$

\section{Exact Boundary Control Problem: Given}

$$
U_{z}(x)=\left\{\begin{array}{l}
\left(u_{z}^{-}(x), v_{z}^{-}(x)\right) \in C^{1}([-1,0]) \times C^{1}([-1,0]), \\
\left(u_{z}^{+}(x), v_{z}^{+}(x)\right) \in C^{1}([0,1]) \times C^{1}([0,1]),
\end{array} \quad z=0, T,\right.
$$

can we find a time $T>0$ and control inputs $h_{1}(t), h_{2}(t)$ in the class of piecewise $C^{1}$ functions defined on $[0, T]$, such that the boundary control system (1.1), (1.9) has a piecewise $C^{1}$ solution $U=U(t, x)$ containing contact discontinuities and satisfying the initial condition (1.10) and the terminal condition

$$
U(T, x)=U_{T}(x) ?
$$


Kong [5] proves the following theorem.

Theorem A Under the hypotheses $\left(H_{1}\right)-\left(H_{4}\right)$, for given $U_{z}(x)(z=0, T)$ (see (1.12)) and for any $T>\bar{T}_{0}$, there exist piecewise $C^{1}$ control inputs $h_{1}(t)$ and $h_{2}(t)$ defined for $t \in[0, T]$ such that system (1.1), (1.9) possesses a piecewise $C^{1}$ solution $U=U(t, x)$ on the domain

$$
\mathcal{D}(T)=\{(t, x) \mid 0 \leq t \leq T,-1 \leq x \leq 1\}
$$

containing 4 contact discontinuities and satisfying

$$
U(0, x)=U_{0}(x), \quad U(T, x)=U_{T}(x), \quad \forall x \in[-1,1],
$$

where $\bar{T}_{0}$ is defined by

$$
\bar{T}_{0}=\max \left\{-\frac{2}{\bar{\lambda}_{1}}, \frac{2}{\underline{\lambda}_{2}}\right\}+\max \left\{-\frac{2}{\bar{\lambda}_{1}}, \frac{2}{\underline{\lambda}_{2}}, \frac{4}{\underline{\lambda}_{2}-\bar{\lambda}_{1}}\right\},
$$

in which

$$
\bar{\lambda}_{1}=\max _{\left|R_{2}\right| \leq M} \mu_{1}\left(R_{2}\right), \quad \underline{\lambda}_{2}=\min _{\left|R_{1}\right| \leq M} \mu_{2}\left(R_{1}\right)
$$

here $M$ is given by

$$
M=\max _{\substack{z=0, T \\ i=1,2}}\left\{\| R_{i}\left(u_{z}^{-}(x), v_{z}^{-}(x)\left\|_{C^{0}([-1,0])},\right\| R_{i}\left(u_{z}^{+}(x), v_{z}^{+}(x) \|_{C^{0}([0,1])}\right\} .\right.\right.
$$

Remark 1.2 Theorem A shows that the system (1.1) with nonlinear boundary conditions (1.9) is globally exactly boundary controllable in the class of piecewise $C^{1}$ functions. However, the control time $\bar{T}_{0}$, defined by (1.16), is not optimal.

In this paper, by a new constructive method, we reprove the global exact boundary controllability of the system (1.1) with the optimal control time. The main result is the following theorem.

Theorem 1.1 Under the hypotheses $\left(H_{1}\right)-\left(H_{4}\right)$, for given $U_{z}(x)(z=0, T)($ see (1.12)) and for any $T>T_{0}$, there exist piecewise $C^{1}$ control inputs $h_{1}(t)$ and $h_{2}(t)$ defined for $t \in[0, T]$ such that system (1.1), (1.9) possesses a piecewise $C^{1}$ solution $U=U(t, x)$, containing 4 contact discontinuities and satisfying (1.15), on the domain $\mathcal{D}(T)$, where $T_{0}$ is defined by

$$
T_{0}=\max \left\{-\frac{2}{\bar{\lambda}_{1}}, \frac{2}{\underline{\lambda}_{2}}, \frac{4}{\underline{\lambda}_{2}-\bar{\lambda}_{1}}\right\} .
$$

Remark 1.3 Comparing (1.16) with (1.19), we observe that $\bar{T}_{0}>T_{0}$. The condition $T>T_{0}$ in Theorem 1.1 is sharp and $T_{0}$ is optimal in the sense that, if $T \leq T_{0}$, we may find a pair of initial and terminal states such that no matter what control inputs we choose, the system will not go from 
the given initial state to the desired terminal state during the time interval $[0, T]$. In this sense, $T_{0}$ defined by (1.19) is called the optimal control time of the system (1.1).

Remark 1.4 The hypothesis $\left(\mathrm{H}_{3}\right)$ is a technical assumption only for constructing contact discontinuities, Kong [5] gives two examples to show that some physical systems always satisfy it. Moreover, if the initial and terminal data are $C^{1}$ smooth, then the hypothesis $\left(H_{3}\right)$ is not needed. In this case, Theorem 1.1 is nothing but the result given in Li and Zhang [8].

The paper is organized as follows. Theorem 1.1 will be proved in Section 2 by a new constructive method. Section 3 gives some important remarks as well as a new application to extremal surfaces in the $(1+n)$-dimensional Minkowski space.

\section{$2 \quad$ Proof of Theorem 1.1}

For readers' convenience, before starting the proof of Theorem 1.1, we first recall the definition of contact discontinuity.

Definition 2.1 $U=U(t, x)$ is called a piecewise $C^{1}$ solution containing a $k$-th $(k=1,2)$ contact discontinuity $x=x_{k}(t)$, if $U=U(t, x)$ satisfies the system (1.1) out of $x=x_{k}(t)$ in the classical sense and satisfies the Rankine-Hugoniot condition on $x=x_{k}(t)$, i.e.,

$$
\begin{gathered}
\sigma \cdot[U]=[F], \\
\sigma=\lambda_{k}\left(U^{+}\right)=\lambda_{k}\left(U^{-}\right),
\end{gathered}
$$

where $U^{ \pm}=U\left(t, x_{k}(t) \pm 0\right)$ and $\sigma=x_{k}^{\prime}(t)$.

Let

$$
\begin{array}{llll}
A=(0,-1), & B=(0,1), & C=(T, 1), & D=(T,-1), \\
O=(0,0), & N=(T, 0), & E=\left(t_{e}, x_{e}\right), & F=\left(t_{f}, x_{f}\right),
\end{array}
$$

where $E$ is the intersection point of the lines

$$
L_{1}: x=\bar{\lambda}_{1} t+1, \quad \text { and } \quad L_{2}: \quad x=\underline{\lambda}_{2} t-1
$$

and $F$ is the intersection point of the lines

$$
\bar{L}_{1}: x=\bar{\lambda}_{1}(t-T)-1 \quad \text { and } \quad \bar{L}_{2}: \quad x=\underline{\lambda}_{2}(t-T)+1 .
$$

Noting (1.19) and $T>T_{0}$, we observe that

$$
t_{e}<t_{f}
$$




\section{Step 1: Generalized Riemann problem (1.1), (1.10)}

First, we solve the generalized Riemann problem for the system (1.1) with discontinuous initial data (1.10). The following lemma comes from [7].

Lemma 2.1 Under the hypotheses $\left(H_{1}\right)-\left(H_{4}\right)$, the generalized Riemann problem (1.1), (1.10) has a unique piecewise $C^{1}$ solution $(u, v)=(u(t, x), v(t, x))$, containing two $C^{1}$ contact discontinuities starting from $O$, on the maximum determined domain $\Omega_{1}$ enclosed by the characteristics $x=$ $x_{1}(t), x=x_{2}(t)$ and the $x$-axis:

$$
\Omega_{1}=\left\{(t, x) \mid 0 \leq t \leq t_{p}, x_{2}(t) \leq x \leq x_{1}(t)\right\},
$$

where $x=x_{1}(t)$ satisfies

$$
\frac{d x_{1}(t)}{d t}=\lambda_{1}(u, v), \quad x_{1}(0)=1,
$$

$x=x_{2}(t)$ satisfies

$$
\frac{d x_{2}(t)}{d t}=\lambda_{2}(u, v), \quad x_{2}(0)=-1,
$$

and while $t_{p}$ is the time coordinate of the intersection point, denoted by $P=\left(t_{p}, x_{p}\right)$, of the characteristic $x=x_{1}(t)$ with the characteristic $x=x_{2}(t)$. See Figure 1.

The solution $(u, v)$ of the generalized Riemann problem (1.1), (1.10) is denoted by $U=U_{1}(t, x)$ on the domain $\Omega_{1}$, its two contact discontinuities are denoted by $x=\xi_{1}(t)$ and $x=\xi_{2}(t)$ respectively. By the definition of contact discontinuity, $x=\xi_{i}(t)(i=1,2)$ satisfy

$$
\frac{\xi_{i}(t)}{d t}=\lambda_{i}\left(U_{1}^{ \pm}\right), \quad \xi_{i}(0)=0 \quad(i=1,2) .
$$

Moreover, let $P_{1}=\left(t_{p_{1}}, x_{p_{1}}\right)$ (resp. $\left.P_{2}=\left(t_{p_{2}}, x_{p_{2}}\right)\right)$ be the the intersection point of the characteristic $x=x_{2}(t)\left(\right.$ resp. $\left.x=x_{1}(t)\right)$ with the contact discontinuity $x=\xi_{1}(t)\left(\right.$ resp. $\left.x=\xi_{2}(t)\right)$. See Figure 1.

\section{Step 2: Backward generalized Riemann problem (1.1), (1.13)}

Similar to Step 1, we can solve the backward generalized Riemann problem for the system (1.1) with discontinuous initial data (1.13). We have.

Lemma 2.2 Under the hypotheses $\left(H_{1}\right)-\left(H_{4}\right)$, the backward generalized Riemann problem (1.1), (1.13) has a unique piecewise $C^{1}$ solution $(u, v)=(u(t, x), v(t, x))$, containing two $C^{1}$ contact discontinuities starting from $N$, on the maximum determined domain $\Omega_{2}$ enclosed by the characteristics $x=\tilde{x}_{1}(t), x=\tilde{x}_{2}(t)$ and the line $t=T$ :

$$
\Omega_{2}=\left\{(t, x) \mid t_{q} \leq t \leq T, \tilde{x}_{1}(t) \leq x \leq \tilde{x}_{2}(t)\right\},
$$


where $x=\tilde{x}_{1}(t)$ satisfies

$$
\left.\frac{d \tilde{x}_{1}(t)}{d t}=\lambda_{1}(u, v)\right), \quad \tilde{x}_{1}(T)=-1
$$

$x=x_{2}(t)$ satisfies

$$
\left.\frac{d \tilde{x}_{2}(t)}{d t}=\lambda_{2}(u, v)\right), \quad \tilde{x}_{2}(T)=1
$$

and while $t_{q}$ is the time coordinate of the intersection point, denoted by $Q=\left(t_{q}, x_{q}\right)$, of the characteristic $x=\tilde{x}_{1}(t)$ with the characteristic $x=\tilde{x}_{2}(t)$. See Figure 1 .

The solution $(u, v)$ of the backward generalized Riemann problem (1.1), (1.13) is denoted by $U=U_{2}(t, x)$ on the domain $\Omega_{2}$, its two contact discontinuities are denoted by $x=\tilde{\xi}_{1}(t)$ and $x=\tilde{\xi}_{2}(t)$ respectively. By the definition of contact discontinuity, $x=\tilde{\xi}_{i}(t)(i=1,2)$ satisfy

$$
\frac{\tilde{\xi}_{i}(t)}{d t}=\lambda_{i}\left(U_{2}^{ \pm}\right), \quad \tilde{\xi}_{i}(0)=0 \quad(i=1,2) .
$$

Moreover, let $Q_{1}=\left(t_{q_{1}}, x_{q_{1}}\right)$ (resp. $Q_{2}=\left(t_{q_{2}}, x_{q_{2}}\right)$ ) be the the intersection point of the characteristic $x=\tilde{x}_{1}(t)\left(\operatorname{resp} . x=\tilde{x}_{2}(t)\right)$ with the contact discontinuity $x=\tilde{\xi}_{2}(t)\left(\operatorname{resp} . x=\tilde{\xi}_{1}(t)\right)$. See Figure 1.

Remark 2.1 From the Steps 1-2, we observe that

$$
0 \leq t_{p} \leq t_{e}, \quad t_{f} \leq t_{q} \leq T
$$

\section{Step 3: Mixed initial-boundary value problem for the system (1.1)}

Let $\Omega_{3}$ be the domain enclosed by the characteristics $P P_{2}$ and $Q Q_{2}$, and the straight line segments $P Q$ and $P_{2} Q_{2}$. It is easy to see that the straight line $P Q$ can be expressed by

$$
x=x_{p}+\alpha\left(t-t_{p}\right) \triangleq c(t), \quad t \in\left[t_{p}, t_{q}\right]
$$

where $\alpha$ is the slope of the line $P Q$

$$
\alpha=\left(x_{q}-x_{p}\right) /\left(t_{q}-t_{p}\right) .
$$

Similarly, the straight line $P_{2} Q_{2}$ can be expressed by

$$
x=x_{p_{2}}+\alpha_{2}\left(t-t_{p_{2}}\right) \triangleq c_{2}(t), \quad t \in\left[t_{p_{2}}, t_{q_{2}}\right],
$$

where $\alpha_{2}$ is the slope of the line $P_{2} Q_{2}$

$$
\alpha_{2}=\left(x_{q_{2}}-x_{p_{2}}\right) /\left(t_{q_{2}}-t_{p_{2}}\right) .
$$


Noting (1.7), the system (1.1) can be equivalently rewritten as

$$
\left\{\begin{array}{l}
\frac{\partial R_{1}}{\partial x}+\frac{1}{\mu_{1}\left(R_{2}\right)} \frac{\partial R_{1}}{\partial t}=0 \\
\frac{\partial R_{2}}{\partial x}+\frac{1}{\mu_{2}\left(R_{1}\right)} \frac{\partial R_{2}}{\partial x}=0
\end{array}\right.
$$

for smooth solutions.

Consider the mixed initial-boundary value problem for the system (2.20) (equivalently, (1.1)) on the domain $\Omega_{3}$ with the boundary conditions

on the characteristic $P P_{2}: x=x_{1}(t) \quad\left(t \in\left[t_{p_{2}}, t_{p}\right]\right)$

$$
R_{2}=R_{2}\left(U_{1}\left(t, x_{1}(t)\right)\right) \triangleq r_{2}(t), \quad t \in\left[t_{p_{2}}, t_{p}\right]
$$

on the characteristic $Q Q_{2}: x=\tilde{x}_{2}\left(t \in\left[t_{q}, t_{q_{2}}\right]\right)$

$$
R_{1}=R_{1}\left(U_{2}\left(t, \tilde{x}_{2}(t)\right)\right) \triangleq \tilde{r}_{1}(t), \quad t \in\left[t_{q}, t_{q_{2}}\right]
$$

and the initial condition on the line segment $P Q$

$$
R_{1}=s_{1}(t), \quad R_{2}=s_{2}(t), \quad t \in\left[t_{p}, t_{q}\right]
$$

where $s_{1}(t), s_{2}(t)$ are $C^{1}$ functions of $t \in\left[t_{p}, t_{q}\right]$. Here we have interchanged the role of $x$ and $t$ variables. In order to ensure the mixed initial-boundary value problem (2.20)-(2.23) have a $C^{1}$ solution on $\Omega_{3}$, the initial data $\left(s_{1}(t), s_{2}(t)\right)$ must satisfy certain compatibility conditions. First of all, it is required that

$$
s_{1}\left(t_{p}\right)=R_{1}\left(U_{1}\left(t_{p}, x_{p}\right)\right), \quad s_{2}\left(t_{q}\right)=R_{2}\left(U_{2}\left(t_{q}, x_{q}\right)\right) .
$$

Moreover, it also require that

$$
s_{1}\left(t_{q}\right)=R_{1}\left(U_{2}\left(t_{q}, x_{q}\right)\right)=\tilde{r}_{1}\left(t_{q}\right), \quad s_{2}\left(t_{p}\right)=R_{2}\left(U_{1}\left(t_{p}, x_{p}\right)\right)=r_{2}\left(t_{p}\right) .
$$

Notice that along the characteristic $Q Q_{2}: x=\tilde{x}_{2}(t)$

$$
\tilde{r}_{1}^{\prime}(t)=\frac{\partial R_{1}}{\partial t}+\mu_{2}\left(R_{1}\right) \frac{\partial R_{1}}{\partial x}=\left(\mu_{2}\left(R_{1}\right)-\mu_{1}\left(R_{2}\right)\right) \frac{\partial R_{1}}{\partial x} .
$$

Then,

$$
\tilde{r}_{1}^{\prime}\left(t_{q}\right)=\left(\mu_{2}\left(R_{1}\left(U_{2}\left(t_{q}, x_{q}\right)\right)\right)-\mu_{1}\left(R_{2}\left(U_{2}\left(t_{q}, x_{q}\right)\right)\right)\right) \frac{\partial R_{1}}{\partial x}\left(t_{q}, x_{q}\right) .
$$

On the other hand, along the line $P Q: x=c(t)$

$$
s_{1}^{\prime}(t)=\frac{\partial R_{1}}{\partial t}+\alpha \frac{\partial R_{1}}{\partial x}=\left(\alpha-\mu_{1}\left(s_{2}\right)\right) \frac{\partial R_{1}}{\partial x} .
$$


Then,

$$
s_{1}^{\prime}\left(t_{q}\right)=\left(\alpha-\mu_{1}\left(s_{2}\left(t_{q}\right)\right)\right) \frac{\partial R_{1}}{\partial x}\left(t_{q}, x_{q}\right)
$$

Therefore we need that

$$
s_{1}^{\prime}\left(t_{q}\right)=\frac{\left(\alpha-\mu_{1}\left(R_{2}\left(U_{2}\left(t_{q}, x_{q}\right)\right)\right)\right)}{\left(\mu_{2}\left(R_{1}\left(U_{2}\left(t_{q}, x_{q}\right)\right)\right)-\mu_{1}\left(R_{2}\left(U_{2}\left(t_{q}, x_{q}\right)\right)\right)\right)} \tilde{r}_{1}^{\prime}\left(t_{q}\right) .
$$

Similarly, at $\left(t_{p}, x_{p}\right)$ we require that

$$
s_{2}^{\prime}\left(t_{p}\right)=\frac{\left(\alpha-\mu_{2}\left(R_{1}\left(U_{1}\left(t_{p}, x_{p}\right)\right)\right)\right)}{\left(\mu_{1}\left(R_{2}\left(U_{1}\left(t_{p}, x_{p}\right)\right)\right)-\mu_{2}\left(R_{1}\left(U_{1}\left(t_{p}, x_{p}\right)\right)\right)\right)} r_{2}^{\prime}\left(t_{p}\right) .
$$

On the other hand, we have

Proposition 2.1 The angles formed by the line segment $P Q$ and the characteristic $Q C$, by the line segment $P Q$ and the characteristic $P B$ are less than $\pi$.

Proof. Consider the angle between $P Q$ and $Q C$. The worst case is given by

$$
P=\left(\frac{2}{\underline{\lambda}_{2}-\underline{\lambda}_{1}}, \frac{\underline{\lambda}_{1}+\underline{\lambda}_{2}}{\underline{\lambda}_{2}-\underline{\lambda}_{1}}\right), \quad Q=\left(T-\frac{2}{\underline{\lambda}_{2}-\underline{\lambda}_{1}},-\frac{\underline{\lambda}_{1}+\underline{\lambda}_{2}}{\underline{\lambda}_{2}-\underline{\lambda}_{1}}\right) .
$$

In the worst case, the slope of $P Q$ is

$$
\frac{d x}{d t}=\frac{2\left(\underline{\lambda}_{1}+\underline{\lambda}_{2}\right)}{4-T\left(\underline{\lambda}_{2}-\underline{\lambda}_{1}\right)}
$$

In order to ensure the angle formed by the line segment $P Q$ and the characteristic $Q C$ is less than $\pi$, it is sufficient to require that the slope of the line segment $P Q$ is less than the slope of the characteristic $Q C$, i.e.,

$$
\frac{2\left(\underline{\lambda}_{1}+\underline{\lambda}_{2}\right)}{4-T\left(\underline{\lambda}_{2}-\underline{\lambda}_{1}\right)}<\underline{\lambda}_{2}
$$

Noting (1.19) and $T>T_{0}$, we have

$$
T>\frac{4}{\underline{\lambda}_{2}-\bar{\lambda}_{1}}
$$

Hence, in order to guarantee the validity of inequality (2.30), it suffices to require that

$$
T>\frac{2}{\underline{\lambda}_{2}}
$$

This is true because of (1.19) and the fact $T>T_{0}$.

Similarly, consider the angle between $P Q$ and $P B$. The worst case is given by

$$
P=\left(\frac{2}{\bar{\lambda}_{2}-\bar{\lambda}_{1}}, \frac{\bar{\lambda}_{1}+\bar{\lambda}_{2}}{\bar{\lambda}_{2}-\bar{\lambda}_{1}}\right), \quad Q=\left(T-\frac{2}{\bar{\lambda}_{2}-\bar{\lambda}_{1}},-\frac{\bar{\lambda}_{1}+\bar{\lambda}_{2}}{\bar{\lambda}_{2}-\bar{\lambda}_{1}}\right) .
$$


A similar argument yields that

$$
T>-\frac{2}{\bar{\lambda}_{1}}
$$

This is also true because of (1.19) and the fact $T>T_{0}$. Thus, the proof of Proposition 2.1 is completed.

Therefore, using Lemma 2.3 and Remark 2.1 in [8], we obtain

Lemma 2.3 Under the hypotheses $\left(H_{1}\right)-\left(H_{4}\right)$, the mixed initial-boundary value problem (2.20)(2.23) admits a unique $C^{1}$ solution $\left(R_{1}, R_{2}\right)=\left(R_{1}(t, x), R_{2}(t, x)\right)$ on $\Omega_{3}$, provided that the compatibility conditions (2.24)-(2.25) and (2.28)-(2.29) hold.

By Lemma 2.3, let $U=U_{3}(t, x)$ be the solution of the system $(1.1)$ corresponding to $\left(R_{1}(t, x), R_{2}(t, x)\right)$ given by Lemma 2.3 on the domain $\Omega_{3}$.

\section{Step 4: Mixed initial-boundary value problem for the system (1.1) - Continuous}

Let $\Omega_{4}$ be the domain enclosed by the characteristics $P P_{1}$ and $Q Q_{1}$, and the straight line segment $P Q: x=c(t)=x_{p}+\alpha\left(t-t_{p}\right), t_{p} \leq t \leq t_{q}$ and the straight line segment $P_{1} Q_{1}$ :

$$
x=x_{p_{1}}+\alpha_{1}\left(t-t_{p_{1}}\right) \triangleq c_{1}(t), \quad t \in\left[t_{p_{1}}, t_{q_{1}}\right]
$$

where $\alpha_{1}$ is the slope of the line $P_{1} Q_{1}$

$$
\alpha_{1}=\left(x_{q_{1}}-x_{p_{1}}\right) /\left(t_{q_{1}}-t_{p_{1}}\right) .
$$

On the domain $\Omega_{4}$, we consider the mixed initial-boundary value problem for the system $(2.20)$ (equivalently, (1.1)) with the boundary conditions

on the characteristic $P P_{1}: x=x_{2}(t) \quad\left(t \in\left[t_{p_{1}}, t_{p}\right]\right)$

$$
R_{1}=R_{1}\left(U_{1}\left(t, x_{2}(t)\right)\right) \triangleq r_{1}(t), \quad t \in\left[t_{p_{1}}, t_{p}\right]
$$

on the characteristic $Q Q_{1}: x=\tilde{x}_{1}(t) t \in\left[t_{q}, t_{q_{1}}\right]$

$$
R_{2}=R_{2}\left(U_{2}\left(t, \tilde{x}_{1}(t)\right)\right) \triangleq \tilde{r}_{2}(t), \quad t \in\left[t_{q}, t_{q_{1}}\right]
$$

and the initial condition on the line segment $P Q$

$$
R_{1}=s_{1}(t), \quad R_{2}=s_{2}(t), \quad t \in\left[t_{p}, t_{q}\right]
$$

As Step 3, we choose $s_{1}(t), s_{2}(t)$ to satisfy the compatibility conditions (2.28)-(2.29) and

$$
\begin{aligned}
& s_{1}^{\prime}\left(t_{p}\right)=\frac{\left(\alpha-\mu_{1}\left(R_{2}\left(U_{1}\left(t_{p}, x_{p}\right)\right)\right)\right)}{\left(\mu_{2}\left(R_{1}\left(U_{1}\left(t_{p}, x_{p}\right)\right)\right)-\mu_{1}\left(R_{2}\left(U_{1}\left(t_{p}, x_{p}\right)\right)\right)\right)} r_{1}^{\prime}\left(t_{p}\right), \\
& s_{2}^{\prime}\left(t_{q}\right)=\frac{\left(\alpha-\mu_{2}\left(R_{1}\left(U_{2}\left(t_{q}, x_{q}\right)\right)\right)\right)}{\left(\mu_{1}\left(R_{2}\left(U_{2}\left(t_{q}, x_{q}\right)\right)\right)-\mu_{2}\left(R_{1}\left(U_{2}\left(t_{q}, x_{q}\right)\right)\right)\right)} \tilde{r}_{2}^{\prime}\left(t_{q}\right) .
\end{aligned}
$$


On the other hand, similar to Proposition 2.1, we have

Proposition 2.2 The angles formed by the line segment $P Q$ and the characteristic $Q D$, by the line segment $P Q$ and the characteristic $P A$ are less than $\pi$.

Therefore, using Lemma 2.3 and Remark 2.1 in [8] again, we have

Lemma 2.4 Under the hypotheses $\left(H_{1}\right)-\left(H_{4}\right)$, the mixed initial-boundary value problem (2.20), (2.32)-(2.34) admits a unique $C^{1}$ solution $\left(R_{1}, R_{2}\right)=\left(R_{1}(t, x), R_{2}(t, x)\right)$ on $\Omega_{4}$, provided that the compatibility conditions (2.24)-(2.25), (2.28)-(2.29) and (2.35) hold.

Similar to Step 3, we denote the solution of the system (1.1) corresponding to $\left(R_{1}(t, x), R_{2}(t, x)\right)$, given by Lemma 2.4, on the domain $\Omega_{4}$ by $U=U_{4}(t, x)$.

\section{Step 5: Cauchy problem for the system (2.20)}

Consider the Cauchy problem in the $x$-direction for the system (2.20) with the following initial condition on the line segment $P_{2} Q_{2}$ :

$$
\begin{aligned}
& R_{1}\left(t, c_{2}(t)\right)=R_{1}\left(U_{3}\left(t, c_{2}(t)\right)\right) \triangleq \sigma_{1}(t), \quad t \in\left[t_{p_{2}}, t_{q_{2}}\right] . \\
& R_{2}\left(t, c_{2}(t)\right)=R_{2}\left(U_{3}\left(t, c_{2}(t)\right)\right) \triangleq \sigma_{2}(t),
\end{aligned}
$$

Similar to Proposition 2.1, we have

Proposition 2.3 The angles formed by the line segment $P_{2} Q_{2}$ and the characteristic $Q_{2} C$, by the line segment $P_{2} Q_{2}$ and the characteristic $P_{2} B$ are less than $\pi$.

Proof. Consider the angle between $P_{2} Q_{2}$ and $Q_{2} C$. The worst case is given by

$$
P_{2}=\left(\frac{1}{\underline{\lambda}_{2}-\underline{\lambda}_{1}}, \frac{\underline{\lambda}_{2}}{\underline{\lambda}_{2}-\underline{\lambda}_{1}}\right), \quad Q_{2}=\left(T-\frac{1}{\underline{\lambda}_{2}-\underline{\lambda}_{1}},-\frac{\underline{\lambda}_{1}}{\underline{\lambda}_{2}-\underline{\lambda}_{1}}\right) .
$$

In the worst case, the slope of $P_{2} Q_{2}$ is

$$
\frac{d x}{d t}=\frac{\underline{\lambda}_{1}+\underline{\lambda}_{2}}{2-T\left(\underline{\lambda}_{2}-\underline{\lambda}_{1}\right)} .
$$

In order to ensure the angle formed by the line $P_{2} Q_{2}$ and the characteristic $Q_{2} C$ is less than $\pi$, it is sufficient to require that the slope of the line $P_{2} Q_{2}$ is less than the slope of the characteristic $Q_{2} C$, i.e.,

$$
\frac{\underline{\lambda}_{1}+\underline{\lambda}_{2}}{2-T\left(\underline{\lambda}_{2}-\underline{\lambda}_{1}\right)}<\underline{\lambda}_{2}
$$

Noting (1.19) and $T>T_{0}$, we have

$$
T>\frac{4}{\underline{\lambda}_{2}-\bar{\lambda}_{1}}
$$


Hence, in order to guarantee the validity of inequality (2.37), it suffices to require that

$$
T>\frac{1}{\underline{\lambda}_{2}} .
$$

Of course, this is true because of (1.19) and the fact $T>T_{0}$.

Similarly, consider the angle between $P_{2} Q_{2}$ and $P_{2} B$. The worst case is given by

$$
P_{2}=\left(\frac{1}{\bar{\lambda}_{2}-\bar{\lambda}_{1}}, \frac{\bar{\lambda}_{2}}{\bar{\lambda}_{2}-\bar{\lambda}_{1}}\right), \quad Q_{2}=\left(T-\frac{1}{\bar{\lambda}_{2}-\bar{\lambda}_{1}},-\frac{\bar{\lambda}_{1}}{\bar{\lambda}_{2}-\bar{\lambda}_{1}}\right) .
$$

A similar argument gives

$$
T>-\frac{1}{\bar{\lambda}_{1}}
$$

Obviously, this is true because of (1.19) and the fact $T>T_{0}$. Thus, the proof of Proposition 2.3 is finished.

Using the Corollary 2.1 in [8], we have

Lemma 2.5 Under the hypotheses $\left(H_{1}\right)-\left(H_{4}\right)$, the Cauchy problem (2.20), (2.36) admits a unique $C^{1}$ solution $\left(R_{1}, R_{2}\right)=\left(R_{1}(t, x), R_{2}(t, x)\right)$ on the maximum determined domain $\Omega_{5}$ enclosed by the straight line segment $P_{2} Q_{2}$, the straight line segment $P_{3} Q_{3}$, the characteristic $P_{2} P_{3}: x=$ $\zeta_{2}(t)\left(t \in\left[t_{p_{2}}, t_{p_{3}}\right]\right)$ and the characteristic $Q_{2} Q_{3}: x=\tilde{\zeta}_{1}(t)\left(t \in\left[t_{q_{3}}, t_{q_{2}}\right]\right)$, where $P_{3}=\left(t_{p_{3}}, 1\right)$ is the intersection point of the characteristic $x=\zeta_{2}(t)$ with the line $B C, Q_{3}=\left(t_{q_{3}}, 1\right)$ is the intersection point of the characteristic $x=\tilde{\zeta}_{1}(t)$ with the line $B C$, while the characteristic $\zeta=\tilde{\zeta}_{1}(t)$ satisfies

$$
\frac{d \tilde{\zeta}_{1}(t)}{d t}=\mu_{1}\left(R_{2}\right), \quad \tilde{\zeta}_{1}\left(t_{q_{2}}\right)=x_{q_{2}}
$$

and the characteristic $\zeta=\zeta_{2}(t)$ satisfies

$$
\frac{d \zeta_{2}(t)}{d t}=\mu_{2}\left(R_{1}\right), \quad \zeta_{2}\left(t_{p_{2}}\right)=x_{p_{2}} .
$$

See Figure 1.

We denote the solution of the system (1.1) corresponding to $\left(R_{1}(t, x), R_{2}(t, x)\right)$, given by Lemma 2.5 , on the domain $\Omega_{5}$ by $U=U_{5}(t, x)$.

Similarly, we consider the Cauchy problem in the anti-x-direction for the system (2.20) with the following initial condition on the line segment $P_{1} Q_{1}$ :

$$
\begin{aligned}
& R_{1}\left(t, c_{1}(t)\right)=R_{1}\left(U_{4}\left(t, c_{1}(t)\right)\right) \triangleq \theta_{1}(t), \quad t \in\left[t_{p_{1}}, t_{q_{1}}\right] . \\
& R_{2}\left(t, c_{1}(t)\right)=R_{2}\left(U_{4}\left(t, c_{1}(t)\right) \triangleq \theta_{2}(t),\right.
\end{aligned}
$$

Similar to Proposition 2.3, we have 
Proposition 2.4 The angles formed by the line segment $P_{1} Q_{1}$ and the characteristic $Q_{1} D$, by the line segment $P_{1} Q_{1}$ and the characteristic $P_{1} A$ are less than $\pi$.

By the Corollary 2.1 in [8], we have

Lemma 2.6 Under the hypotheses $\left(H_{1}\right)-\left(H_{4}\right)$, the Cauchy problem (2.20), (2.40) admits a unique $C^{1}$ solution $\left(R_{1}, R_{2}\right)=\left(R_{1}(t, x), R_{2}(t, x)\right)$ on the maximum determined domain $\Omega_{6}$ enclosed by the straight line segment $P_{1} Q_{1}$, the straight line segment $P_{4} Q_{4}$, the characteristic $P_{1} P_{4}: x=\zeta_{1}(t)(t \in$ $\left.\left[t_{p_{1}}, t_{p_{4}}\right]\right)$ and the characteristic $Q_{1} Q_{4}: x=\tilde{\zeta}_{2}(t)\left(t \in\left[t_{q_{4}}, t_{q_{1}}\right]\right)$ (see Fig. 1), where $P_{4}=\left(t_{p_{4}}, 1\right)$ is the intersection point of the characteristic $\zeta=\zeta_{1}(t)$ with the line $A D, Q_{4}=\left(t_{q_{4}}, 1\right)$ is the intersection point of the characteristic $\zeta=\tilde{\zeta}_{2}(t)$ with the line $A D$, while the characteristic $\zeta=\zeta_{1}(t)$ satisfies

$$
\frac{d \zeta_{1}(t)}{d t}=\mu_{1}\left(R_{2}\right), \quad \zeta_{1}\left(t_{p_{1}}\right)=x_{p_{1}}
$$

and the characteristic $\zeta=\tilde{\zeta}_{2}(t)$ satisfies

$$
\frac{d \tilde{\zeta}_{2}(t)}{d t}=\mu_{2}\left(R_{1}\right), \quad \tilde{\zeta}_{2}\left(t_{q_{1}}\right)=x_{q_{1}}
$$

See Figure 1.

Let $U=U_{6}(t, x)$ be the solution of the system (1.1) corresponding to $\left(R_{1}(t, x), R_{2}(t, x)\right)$, given by Lemma 2.6 , on the domain $\Omega_{6}$.

\section{Step 6: Goursat problem for the system (2.20)}

We next consider the Goursat problem in the $x$-direction for the system (2.20) with the following characteristic boundary conditions:

on the characteristic $P_{2} B: x=x_{1}(t)\left(t \in\left[0, t_{p_{2}}\right]\right)$

$$
R_{2}=R_{2}\left(U_{1}\left(t, x_{1}(t)\right)\right) \triangleq \eta_{2}(t), \quad t \in\left[0, t_{p_{2}}\right]
$$

on the characteristic $P_{2} P_{3}: x=\zeta_{2}(t)\left(t \in\left[t_{p_{2}}, t_{p_{3}}\right]\right)$

$$
R_{1}=R_{1}\left(U_{5}\left(t, \zeta_{2}(t)\right)\right) \triangleq \eta_{1}(t), \quad t \in\left[t_{p_{2}}, t_{p_{3}}\right]
$$

By the Lemma 2.2 in $[8]$, we have

Lemma 2.7 Under the hypotheses $\left(H_{1}\right)-\left(H_{4}\right)$, the Goursat problem (2.20), (2.43)-(2.44) has a unique $C^{1}$ solution $\left(R_{1}, R_{2}\right)=\left(R_{1}(t, x), R_{2}(t, x)\right)$ on the domain $\Omega_{7}$ enclosed by the straight 
line segment $B P_{3}$, the characteristic $B P_{2}: x=x_{1}(t)\left(t \in\left[0, t_{p_{2}}\right]\right)$ and the characteristic $P_{2} P_{3}$ : $x=\zeta_{2}(t)\left(t \in\left[t_{p_{2}}, t_{p_{3}}\right]\right)$. See Figure 1. Moreover, it holds that

$$
\begin{array}{ll}
R_{1}\left(t, \zeta_{2}(t)\right)=\eta_{1}(t), & \forall t \in\left[t_{p_{2}}, t_{p_{3}}\right], \\
R_{2}\left(t, x_{1}(t)\right)=\eta_{2}(t), & \forall t \in\left[0, t_{p_{2}}\right] .
\end{array}
$$

Let $U=U_{7}(t, x)$ be the solution of the system (1.1) corresponding to $\left(R_{1}(t, x), R_{2}(t, x)\right)$, given by Lemma 2.7 , on the domain $\Omega_{7}$.

Similarly, consider the Goursat problem in the $x$-direction for the system (2.20) with the following characteristic boundary conditions:

on the characteristic $Q_{2} C: x=\tilde{x}_{2}(t)\left(t \in\left[t_{q_{2}}, T\right]\right)$

$$
R_{1}=R_{1}\left(U_{2}\left(t, \tilde{x}_{2}(t)\right)\right) \triangleq \tilde{\eta}_{1}(t), \quad t \in\left[t_{q_{2}}, T\right]
$$

on the characteristic $Q_{2} Q_{3}: x=\tilde{\zeta}_{1}(t)\left(t \in\left[t_{q_{3}}, t_{q_{2}}\right]\right)$

$$
R_{2}=R_{2}\left(U_{5}\left(t, \tilde{\zeta}_{1}(t)\right)\right) \triangleq \tilde{\eta}_{2}(t), \quad t \in\left[t_{q_{3}}, t_{q_{2}}\right]
$$

Similar to Lemma 2.7 , we have

Lemma 2.8 Under the hypotheses $\left(H_{1}\right)-\left(H_{4}\right)$, the Goursat problem (2.20), (2.46)-(2.47) has a unique $C^{1}$ solution $\left(R_{1}, R_{2}\right)=\left(R_{1}(t, x), R_{2}(t, x)\right)$ on the domain $\Omega_{8}$ enclosed by the straight line segment $Q_{3} C$, the characteristic $Q_{2} C: x=\tilde{x}_{2}(t)\left(t \in\left[t_{q_{2}}, T\right]\right)$ and the characteristic $Q_{2} Q_{3}$ : $x=\tilde{\zeta}_{1}(t)\left(t \in\left[t_{q_{3}}, t_{q_{2}}\right]\right)$. See Figure 1. Moreover, it holds that

$$
\begin{aligned}
& R_{1}\left(t, \tilde{x}_{2}(t)\right)=\tilde{\eta}_{1}(t), \quad \forall t \in\left[t_{q_{2}}, T\right], \\
& R_{2}\left(t, \tilde{\zeta}_{1}(t)\right)=\tilde{\eta}_{2}(t), \quad \forall t \in\left[t_{q_{3}}, t_{q_{2}}\right] .
\end{aligned}
$$

Let $U=U_{8}(t, x)$ be the solution of the system (1.1) corresponding to $\left(R_{1}(t, x), R_{2}(t, x)\right)$, given by Lemma 2.8 , on the domain $\Omega_{8}$.

On the other hand, we consider the Goursat problem in the anti- $x$-direction for the system (2.20) with the following characteristic boundary conditions:

on the characteristic $Q_{1} D: x=\tilde{x}_{1}(t)\left(t \in\left[t_{q_{1}}, T\right]\right)$

$$
R_{2}=R_{2}\left(U_{2}\left(t, \tilde{x}_{1}(t)\right)\right) \triangleq \tilde{\gamma}_{2}(t), \quad t \in\left[t_{q_{1}}, T\right]
$$

on the characteristic $Q_{1} Q_{4}: x=\tilde{\zeta}_{2}(t)\left(t \in\left[t_{q_{4}}, t_{q_{1}}\right]\right)$

$$
R_{1}=R_{1}\left(U_{6}\left(t, \tilde{\zeta}_{2}(t)\right)\right) \triangleq \tilde{\gamma}_{1}(t), \quad t \in\left[t_{q_{4}}, t_{q_{1}}\right] .
$$


Similar to Lemma 2.8, we have

Lemma 2.9 Under the hypotheses $\left(H_{1}\right)-\left(H_{4}\right)$, the Goursat problem (2.20), (2.49)-(2.50) has a unique $C^{1}$ solution $\left(R_{1}, R_{2}\right)=\left(R_{1}(t, x), R_{2}(t, x)\right)$ on the domain $\Omega_{9}$ enclosed by the straight line segment $Q_{4} D$, the characteristic $Q_{1} D: x=\tilde{x}_{1}(t)\left(t \in\left[t_{q_{1}}, T\right]\right)$ and the characteristic $Q_{4} Q_{1}$ : $x=\tilde{\zeta}_{2}(t)\left(t \in\left[t_{q_{4}}, t_{q_{1}}\right]\right)$. See Figure 1. Moreover, it holds that

$$
\begin{aligned}
& R_{1}\left(t, \tilde{\zeta}_{2}(t)\right)=\tilde{\gamma}_{1}(t), \quad \forall t \in\left[t_{q_{4}}, t_{q_{1}}\right] \\
& R_{2}\left(t, \tilde{x}_{1}(t)\right)=\tilde{\gamma}_{2}(t), \quad \forall t \in\left[t_{q_{1}}, T\right] .
\end{aligned}
$$

Let $U=U_{9}(t, x)$ be the solution of the system (1.1) corresponding to $\left(R_{1}(t, x), R_{2}(t, x)\right)$, given by Lemma 2.9 , on the domain $\Omega_{9}$.

Finally, we consider the Goursat problem in the anti- $x$-direction for the system (2.20) with the following characteristic boundary conditions:

on the characteristic $A P_{1}: x=x_{2}(t)\left(t \in\left[0, t_{p_{1}}\right]\right)$

$$
R_{1}=R_{1}\left(U_{1}\left(t, x_{2}(t)\right)\right) \triangleq \gamma_{1}(t), \quad t \in\left[0, t_{p_{1}}\right]
$$

on the characteristic $P_{1} P_{4}: x=\zeta_{1}(t)\left(t \in\left[t_{p_{1}}, t_{p_{4}}\right]\right)$

$$
R_{2}=R_{2}\left(U_{6}\left(t, \zeta_{1}(t)\right)\right) \triangleq \gamma_{1}(t), \quad t \in\left[t_{p_{1}}, t_{p_{4}}\right]
$$

Similar to Lemma 2.9, we have

Lemma 2.10 Under the hypotheses $\left(H_{1}\right)-\left(H_{4}\right)$, the Goursat problem (2.20), (2.52)-(2.53) has a unique $C^{1}$ solution $\left(R_{1}, R_{2}\right)=\left(R_{1}(t, x), R_{2}(t, x)\right)$ on the domain $\Omega_{10}$ enclosed by the straight line segment $A P_{4}$, the characteristic $A P_{1}: x=x_{2}(t)\left(t \in\left[0, t_{p_{1}}\right]\right)$ and the characteristic $P_{1} P_{4}$ : $x=\zeta_{1}(t)\left(t \in\left[t_{p_{1}}, t_{p_{4}}\right]\right)$. See Figure 1. Moreover, it holds that

$$
\begin{aligned}
& R_{1}\left(t, x_{2}(t)\right)=\gamma_{1}(t), \quad \forall t \in\left[0, t_{p_{1}}\right], \\
& R_{2}\left(t, \zeta_{1}(t)\right)=\gamma_{2}(t), \quad \forall t \in\left[t_{p_{1}}, t_{p_{4}}\right] .
\end{aligned}
$$

Let $U=U_{10}(t, x)$ be the solution of the system (1.1) corresponding to $\left(R_{1}(t, x), R_{2}(t, x)\right)$, given by Lemma 2.10 , on the domain $\Omega_{10}$.

\section{Step 7: Piecewise $C^{1}$ solution with four $C^{1}$ contact discontinuities}

Now we choose $s_{1}(t), s_{2}(t)$ in $(2.23)$ from the space $C^{1}\left[t_{p}, t_{q}\right]$ so that the compatibility conditions $(2.24)-(2.25),(2.28)-(2.29)$ and $(2.35)$ are all satisfied and the $C^{0}$ norms of $s_{1}(t), s_{2}(t)$ are bounded by $M$. 
Define the piecewise $C^{1}$ function

$$
U=U(t, x)=\left\{\begin{array}{cc}
U_{1}(t, x), & \text { for }(t, x) \in \Omega_{1}, \\
\ldots & \ldots \\
U_{10}(t, x), & \text { for }(t, x) \in \Omega_{10}
\end{array}\right.
$$

and the piecewise $C^{1}$ curves

$$
\begin{aligned}
& \ell_{1}=\left\{(t, x) \mid x=\xi_{1}(t) \quad \text { for } t \in\left[0, t_{p_{1}}\right] ; \quad x=\zeta_{1}(t) \text { for } t \in\left[t_{p_{1}}, t_{p_{4}}\right]\right\}, \\
& \ell_{2}=\left\{(t, x) \mid x=\xi_{2}(t) \quad \text { for } t \in\left[0, t_{p_{2}}\right] ; \quad x=\zeta_{2}(t) \text { for } t \in\left[t_{p_{2}}, t_{p_{3}}\right]\right\} \text {, } \\
& \tilde{\ell}_{1}=\left\{(t, x) \mid x=\tilde{\xi}_{1}(t) \text { for } t \in\left[t_{q_{2}}, T\right] ; \quad x=\tilde{\zeta}_{1}(t) \text { for } t \in\left[t_{q_{3}}, t_{q_{2}}\right]\right\} \text {, } \\
& \tilde{\ell}_{2}=\left\{(t, x) \mid x=\tilde{\xi}_{2}(t) \text { for } t \in\left[t_{q_{1}}, T\right] ; \quad x=\tilde{\zeta}_{2}(t) \text { for } t \in\left[t_{q_{4}}, t_{q_{1}}\right]\right\} \text {. }
\end{aligned}
$$

By the construction of $U_{i}(i=1, \cdots, 10)$, we observe that $U=U(t, x)$ defined by $(2.55)$ is a $C^{1}$ function out of curves $\ell_{1}, \ell_{2}, \tilde{\ell}_{1}$ and $\tilde{\ell}_{2}$; meanwhile $\ell_{1}$ (i.e., $O P_{4}$ ), $\ell_{2}$ (i.e., $O P_{3}$ ), $\tilde{\ell}_{1}$ (i.e., $N Q_{3}$ ) and $\tilde{\ell}_{2}$ (i.e., $N Q_{4}$ ) are $C^{1}$ smooth curves. See Figure 1.

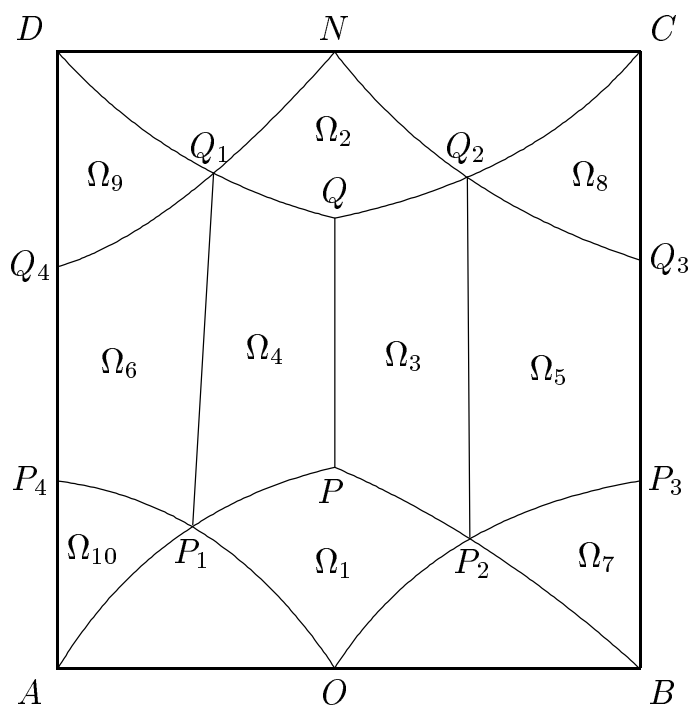

Figure 1: Domains $\Omega_{i}(i=1, \cdots, 10)$; Characteristics: $A P: x=x_{2}(t), B P: x=x_{1}(t), C Q$ : $x=\tilde{x}_{2}(t), D Q: x=\tilde{x}_{1}(t)$; Contact discontinuities: $O P_{1}: x=\xi_{1}(t), P_{1} P_{4}: x=\zeta_{1}(t), O P_{2}:$ $x=\xi_{2}(t), P_{2} P_{3}: x=\zeta_{2}(t), N Q_{1}: x=\tilde{\xi}_{2}(t), Q_{1} Q_{4}: x=\tilde{\zeta}_{2}(t), N Q_{2}: x=\tilde{\xi}_{1}(t), Q_{2} Q_{3}: x=$ $\tilde{\zeta}_{1}(t)$

Obviously, $U=U(t, x)$, defined by (2.55), satisfies the system (1.1) on the domain $\mathcal{D}(T)$, but out of the curves $\ell_{1}, \ell_{2}, \tilde{\ell}_{1}$ and $\tilde{\ell}_{2}$, in the class sense. On the other hand, it is clear that $U=U(t, x)$ satisfies the condition (1.15). 
In what follows, we show that the curves $\ell_{1}$ and $\tilde{\ell}_{1}$ (resp. $\ell_{2}$ and $\tilde{\ell}_{2}$ ) are contact discontinuities corresponding to $\lambda_{1}(U)$ (resp. $\lambda_{1}(U)$ ).

In fact, we only need to prove that the Rankine-Hugoniot conditions (2.1)-(2.2) hold on the curves $x=\zeta_{1}(t)\left(t \in\left[t_{p_{1}}, t_{p_{4}}\right]\right), x=\zeta_{2}(t)\left(t \in\left[t_{p_{2}}, t_{p_{3}}\right]\right), x=\tilde{\zeta}_{1}(t)\left(t \in\left[t_{q_{3}}, t_{q_{2}}\right]\right)$ and $x=\tilde{\zeta}_{2}(t)(t \in$ $\left.\left[t_{q_{4}}, t_{q_{1}}\right]\right)$.

It follows from (1.8) and (2.53) that

$$
\lambda_{1}\left(U_{6}\left(t, \zeta_{1}(t)\right)\right)=\lambda_{1}\left(U_{10}\left(t, \zeta_{1}(t)\right)\right) \triangleq \sigma(t), \quad \forall t \in\left[t_{p_{1}}, t_{p_{4}}\right] .
$$

This is just the desired (2.2) for the case $k=1$ and $x=\zeta_{1}(t)$.

We next show that the Rankine-Hugoniot condition (2.1) holds on $x=\zeta_{1}(t)$.

By $\left(H_{3}\right)$, we know that, for any fixed $t \in\left[t_{p_{1}}, t_{p_{4}}\right]$, there exists a $C^{1}$ curve segment $U=U(\tau)$ $\left(\tau \in\left[\tau_{1}, \tau_{2}\right]\right)$ in $\mathcal{N}$ such that

$$
U\left(\tau_{1}\right)=U_{6}\left(t, \zeta_{1}(t)\right), \quad U\left(\tau_{2}\right)=U_{10}\left(t, \zeta_{1}(t)\right)
$$

and

$$
\lambda_{1}(U(\tau))=\sigma(t), \quad \forall \tau \in\left[\tau_{1}, \tau_{2}\right] .
$$

Differentiating (2.58) with respect to $\tau$ gives

$$
\nabla \lambda_{1}(U(\tau)) \cdot \frac{d U}{d \tau}(\tau)=0, \quad \forall \tau \in\left[\tau_{1}, \tau_{2}\right]
$$

By (1.3), (1.5) and (2.59), we observe that $\frac{d U}{d \tau}(\tau)$ is proportional to $\vec{r}_{2}(U(\tau))$. Then we have

$$
\sigma(t) \frac{d U}{d \tau}(\tau)=\nabla F(U(\tau)) \frac{d U}{d \tau}(\tau), \quad \forall \tau \in\left[\tau_{1}, \tau_{2}\right] .
$$

Integrating this yields the Rankine-Hugoniot condition (2.1) on $x=\zeta_{1}(t)$.

Others are similar.

\section{Step 8: Control inputs $h_{1}(t)$ and $h_{2}(t)$}

Finally, we define $h_{1}(t)$ and $h_{2}(t)$ as follows

$$
h_{1}(t)=\left\{\begin{array}{lll}
-B_{1}\left(U_{10}(t,-1), t\right), & \text { as } & t \in\left[0, t_{p_{4}}\right], \\
-B_{1}\left(U_{6}(t,-1), t\right), & \text { as } & t \in\left[t_{p_{4}}, t_{q_{4}}\right], \\
-B_{1}\left(U_{9}(t,-1), t\right), & \text { as } & t \in\left[t_{q_{4}}, T\right]
\end{array}\right.
$$

and

$$
h_{2}(t)=\left\{\begin{array}{lll}
-B_{2}\left(U_{7}(t, 1), t\right), & \text { as } & t \in\left[0, t_{p_{3}}\right], \\
-B_{2}\left(U_{5}(t, 1), t\right), & \text { as } & t \in\left[t_{p_{3}}, t_{q_{3}}\right], \\
-B_{2}\left(U_{8}(t, 1), t\right), & \text { as } & t \in\left[t_{q_{3}}, T\right] .
\end{array}\right.
$$


$h_{1}(t)$ and $h_{2}(t)$ are just the desired control inputs such that the system (1.1), (1.9) possesses a piecewise $C^{1}$ solution $U=U(t, x)$, containing 4 contact discontinuities and satisfying (1.15), on the domain $\mathcal{D}(T)$, provided that $T>T_{0}$. Thus, the proof of Theorem 1.1 is completed.

\section{Some remarks and a new application}

In this section, we give some important remarks as well as a new application of our theory.

Remark 3.1 Some physical systems always satisfy the hypotheses $\left(H_{1}\right)-\left(H_{4}\right)$, for example, the system of isentropic gas with the Von Kármán-Tsien pressure law, the system of relativistic gas dynamics with the relativistic counterpart of the Chaplygin pressure law, etc. (see [5]).

Remark 3.2 Consider general quasilinear system of conservation laws

$$
\frac{\partial u}{\partial t}+\frac{\partial f(u)}{\partial x}=0
$$

where $u=\left(u_{1}, \cdots, u_{n}\right)^{T}$ is the unknown vector function of $(t, x), f: \mathbb{R}^{n} \rightarrow \mathbb{R}^{n}$ is a given $C^{2}$ vector function of $u$. Suppose that on the domain under consideration, (3.1) is a non-strictly hyperbolic system with two characteristics, and each characteristic has a constant multiplicity, say, on the domain under consideration,

$$
\lambda_{1}(u) \equiv \cdots \equiv \lambda_{m}(u) \triangleq \lambda(u)<\mu(u) \triangleq \lambda_{m+1}(u) \equiv \cdots \equiv \lambda_{n}(u)
$$

where $1 \leq m \leq n-1$. When $m>1$ or $m<n-1$, the system (3.1) is non-strictly hyperbolic. In particular, when $n \geq 4$ and $1<m<n-1$, the system (3.1) is rich, and $\lambda(u), \mu(u)$ must be linearly degenerate (see [9] and [1]-[4]). In this case, the system (3.1) can be rewritten as

$$
\begin{array}{ll}
\frac{\partial R_{i}}{\partial t}+\lambda(u) \frac{\partial R_{i}}{\partial x}=0 & (i=1, \cdots, m), \\
\frac{\partial R_{j}}{\partial t}+\mu(u) \frac{\partial R_{j}}{\partial x}=0 & (j=m+1, \cdots, n),
\end{array}
$$

where $R_{i}(i=1, \cdots, n)$ are Riemann invariants. For the present situation we have a similar result.

Remark 3.3 Hypothesis $\left(H_{3}\right)$ is a geometric assumption for constructing contact discontinuities with non-small jumps. It is needed since we do not require that the oscillations of $u_{0}^{ \pm}(x), v_{0}^{ \pm}(x), u_{T}^{ \pm}(x)$, $v_{T}^{ \pm}(x)$ and the jumps $\left|u_{0}^{+}(0)-u_{0}^{+}(0)\right|,\left|v_{0}^{+}(0)-v_{0}^{+}(0)\right|,\left|u_{T}^{+}(0)-u_{T}^{+}(0)\right|,\left|v_{T}^{+}(0)-v_{T}^{+}(0)\right|$ are small. If the above jumps are small, then the hypothesis $\left(H_{3}\right)$ is not needed. Moreover, in Theorem 1.1, a part of contact discontinuities in the solution $U=U(t, x)$ may disappear. If the initial and terminal functions are continuous at $x=0$, then the contact discontinuities in $U=U(t, x)$ degenerate weak 
discontinuities. If the initial and terminal functions are $C^{1}$ smooth on $[-1,1]$, then the solution $U=U(t, x)$ is also $C^{1}$ smooth, in this case Theorem 1.1 is nothing but the result given in [8].

Remark 3.4 The hypothesis $\left(H_{4}\right)$ is also needful since we do not require that the oscillations and jumps of the initial and terminal functions are small. If the oscillations and jumps of the initial and terminal functions are small, then the hypothesis $\left(H_{4}\right)$ is not needed. Moreover, it is also required in the proof of Theorem 1.1 that the mapping defined by (1.6) is inverse, i.e., we can solve $U$ from (1.6).

\section{Application to the extremal surfaces in $(1+n)$-dimensional Minkowski space-time}

We next give a new application of Theorem 1.1.

Let $(t, x, y)$ be points on $(1+2)$-dimensional Minkowski space-time. A time-like surface takes the form

$$
y=\phi(t, x)
$$

This surface is called to be extremal surface if $\phi$ is the critical point of the area functional

$$
I=\iint \sqrt{1+\phi_{x}^{2}-\phi_{t}^{2}} d x d t
$$

The corresponding Euler-Lagrange equation is as follows

$$
\left(\frac{\phi_{t}}{\sqrt{1+\phi_{x}^{2}-\phi_{t}^{2}}}\right)_{t}-\left(\frac{\phi_{x}}{\sqrt{1+\phi_{x}^{2}-\phi_{t}^{2}}}\right)_{x}=0 .
$$

Notice that, if $\sqrt{1+\phi_{x}^{2}-\phi_{t}^{2}} \neq 0$, then (3.5) is nothing but the Born-Infeld equation (see [2]).

Recently, Brenier [3] suggests an equation for extremal surfaces in the 5-dimensional Minkowski space, which is related to classical Electrodynamics. By prescribing $(t, s) \rightarrow(t, s, X(t, s))$ to be an extremal surface in the 5 -dimensional Minkowski space $\left(t, s, x_{1}, x_{2}, x_{3}\right)$ with the signature $(-,+,+,+,+)$, we know that the area functional is

$$
I=\iint \sqrt{1+\left|\partial_{s} X\right|^{2}-\left|\partial_{t} X\right|^{2}-\left|\partial_{s} X \times \partial_{t} X\right|^{2}} d s d t .
$$

The corresponding Euler-Lagrange equation is

$$
\begin{aligned}
& \left(\frac{\phi_{t}}{\sqrt{1+\left|\phi_{x}\right|^{2}-\left|\phi_{t}\right|^{2}-\left|\phi_{t} \times \phi_{x}\right|^{2}}}\right)_{t}-\left(\frac{\phi_{x}}{\sqrt{1+\left|\phi_{x}\right|^{2}-\left|\phi_{t}\right|^{2}-\left|\phi_{t} \times \phi_{x}\right|^{2}}}\right)_{x}+ \\
& \left(\frac{\phi_{x} \times\left(\phi_{t} \times \phi_{x}\right)}{\sqrt{1+\left|\phi_{x}\right|^{2}-\left|\phi_{t}\right|^{2}-\left|\phi_{t} \times \phi_{x}\right|^{2}}}\right)_{t}-\left(\frac{\phi_{t} \times\left(\phi_{t} \times \phi_{x}\right)}{\sqrt{1+\left|\phi_{x}\right|^{2}-\left|\phi_{t}\right|^{2}-\left|\phi_{t} \times \phi_{x}\right|^{2}}}\right)_{x}=0
\end{aligned}
$$

where $x$ stands for $s$ in (3.6), and $\phi=\left(\phi_{1}, \phi_{2}, \phi_{3}\right)^{T}$ represents $X$ in (3.6). 
More generally, we consider a vector function $\phi=\left(\phi_{1} \cdots, \phi_{n}\right)^{T}$, which is the critical point of the area functional

$$
I=\iint \sqrt{1+\left|\phi_{x}\right|^{2}-\left|\phi_{t}\right|^{2}-\left|\phi_{t}\right|^{2}\left|\phi_{x}\right|^{2}+\left\langle\phi_{t}, \phi_{x}\right\rangle^{2}} d x d t
$$

where $\langle\cdot, \cdot\rangle$ stands for the inner product. The corresponding Euler-Lagrange equation is as follows

$$
\begin{aligned}
& \left(\frac{\phi_{t}}{\sqrt{1+\left|\phi_{x}\right|^{2}-\left|\phi_{t}\right|^{2}-\left|\phi_{t}\right|^{2}\left|\phi_{x}\right|^{2}+\left\langle\phi_{t}, \phi_{x}\right\rangle^{2}}}\right)_{t}{ }_{t}^{-} \\
& \left(\frac{\phi_{x}}{\sqrt{1+\left|\phi_{x}\right|^{2}-\left|\phi_{t}\right|^{2}-\left|\phi_{t}\right|^{2}\left|\phi_{x}\right|^{2}+\left\langle\phi_{t}, \phi_{x}\right\rangle^{2}}}\right)_{x}^{+} \\
& \left(\frac{\left|\phi_{x}\right|^{2} \phi_{t}-\left\langle\phi_{t}, \phi_{x}\right\rangle \phi_{x}}{\sqrt{1+\left|\phi_{x}\right|^{2}-\left|\phi_{t}\right|^{2}-\left|\phi_{t}\right|^{2}\left|\phi_{x}\right|^{2}+\left\langle\phi_{t}, \phi_{x}\right\rangle^{2}}}\right)_{t}- \\
& \left(\frac{\left\langle\phi_{t}, \phi_{x}\right\rangle \phi_{t}-\left|\phi_{t}\right|^{2} \phi_{x}}{\sqrt{1+\left|\phi_{x}\right|^{2}-\left|\phi_{t}\right|^{2}-\left|\phi_{t}\right|^{2}\left|\phi_{x}\right|^{2}+\left\langle\phi_{t}, \phi_{x}\right\rangle^{2}}}\right)_{x}=0 .
\end{aligned}
$$

Remark 3.5 When $n=1$, the equation (3.9) is nothing but the equation (3.5); when $n=3$, the equation (3.9) becomes the equation (3.7).

Let

$$
u=\phi_{x}, \quad v=\phi_{t} .
$$

Then (3.9) can be equivalently rewritten as

$$
\left\{\begin{array}{l}
u_{t}-v_{x}=0, \\
\left(\frac{v}{\sqrt{1+|u|^{2}-|v|^{2}-|v|^{2}|u|^{2}+\langle u, v\rangle^{2}}}\right){ }_{t}^{t} \\
\left(\frac{u}{\sqrt{1+|u|^{2}-|v|^{2}-|v|^{2}|u|^{2}+\langle u, v\rangle^{2}}}\right)_{x}^{+} \\
\left(\frac{|u|^{2} v-\langle u, v\rangle u}{\sqrt{1+|u|^{2}-|v|^{2}-|v|^{2}|u|^{2}+\langle u, v\rangle^{2}}}\right)_{t}^{t} \\
\left(\frac{\langle u, v\rangle v-|v|^{2} u}{\sqrt{1+|u|^{2}-|v|^{2}-|v|^{2}|u|^{2}+\langle u, v\rangle^{2}}}\right)_{x}=0
\end{array}\right.
$$

for smooth solutions. The following lemma comes from [6].

Lemma 3.1 If $\triangle(u, v) \triangleq 1+|u|^{2}-|v|^{2}-|v|^{2}|u|^{2}+\langle u, v\rangle^{2}>0$, then (3.11) is a non-strictly hyperbolic system with two n-constant multiple eigenvalues:

$$
\lambda_{1} \equiv \cdots \equiv \lambda_{n} \triangleq \lambda_{-}<\lambda_{+} \triangleq \lambda_{n+1} \equiv \cdots \equiv \lambda_{2 n}
$$

where

$$
\lambda_{ \pm}=\frac{1}{1+|u|^{2}}\left(-\langle u, v\rangle \pm \sqrt{1+|u|^{2}-|v|^{2}-|v|^{2}|u|^{2}+\langle u, v\rangle^{2}}\right),
$$

moreover, the system (3.11) is linear degenerate. 
Noting Remark 3.2, under suitable assumptions we can obtain the global exact boundary controllability for the system (3.11) (equivalently, the equation (3.9)) in the class of piecewise $C^{1}$ functions. For the limited space, we omit the details.

Acknowledgements. The first author (Kong) thanks Prof. Ta-tsien Li for his constant encouragement and valuable suggestions. This work was completed while Kong was visiting the University of Potsdam during the summer of 2003. Kong thanks Prof. B.-W. Schulze for his invitation and hospitality. This work was supported in part by the National Science Foundation of China under Grant No. 10001024, the Deutsche Forschungsgemeinschaft (DFG), and the Qi Ming Xing programme of Shanghai Government.

\section{References}

[1] G. Boillat, Chocs caractéristiques, C. R. Acad. Sci., Paris, Sér. A 274, 1018-1021 (1972).

[2] M. Born and L. Infeld, Foundation of the new field theory, Proc. Roy. Soc. London A144(1934), 425-451.

[3] Y. Brenier, Some Geometric PDEs Related to Hydrodynamics and Electrodynamics, Proceedings of ICM 2002, 3(2002), 761-772.

[4] H. Freistühler, Linear degeneracy and shock waves, Math. Zeit. 207 (1991), 583-596.

[5] De-Xing Kong, Global exact boundary controllability of a class of quasilinear hyperbolic systems of conservation laws, Systems \& Control Letters 47 (2002), 287-298.

[6] De-Xing Kong and Qing-You Sun, On the equation for extremal surfaces in $(1+n)$-dimensional Minkowski space, to appear.

[7] De-Xing Kong and M. Tsuji, Global solutions for $2 \times 2$ hyperbolic systems with linearly degenerate characteristics, Funkcialaj Ekvacioj 42 (1999), 129-155.

[8] Ta-tsien Li and Bing-yu Zhang, Global exact controllability of a class of quasilinear hyperbolic systems, J. Math. Anal. Appl. 225 (1998), 289-311.

[9] D. Serre, Systems of Conservation Laws 2: Geometric Structures, Oscillations, and InitialBoundary Value Problems, Canmbridge University Press, Cambridge, 2000. 